\title{
Funciones distancia asimétricas y no positivas definidas Parte I: Marco teórico
}

\section{Asymmetric and non-positive definite distance functions Part I: Theoretical framework}

\author{
H. Sánchez-Larios \\ Instituto de Ingeniería, Universidad Nacional Autónoma de México. \\ E-mails: hsanchezl@ii.unam.mx \\ S.T. Guillén-Burguete \\ Instituto de Ingeniería, Universidad Nacional Autónoma de México. \\ E-mails:sguillenb@ii.unam.mx
}

(Recibido: enero de 2006; aceptado: febrero de 2007)

\begin{abstract}
Resumen
Se propone un marco teórico para modelar funciones distancia generalizadas, las cuales pueden ser asimétricas y no positivas definidas. Se da una definición de longitud de arco asociada a una función distancia generalizada $d$. La función distancia $d$ cumple la propiedad de identidad, pero a diferencia de las métricas, puede no satisfacer la desigualdad del triángulo, o las propiedades de simetría y definitoreidad. Mostramos que cada función distancia generalizada $d$ induce ciertos arcos, que llamamos " $d$-inducidos", los cuales cumplen una ley de conservación de la distancia $d$ y son una generalización de los segmentos de línea recta del espacio euclidiano. También se muestra que si $d$ satisface la desigualdad del triángulo, entonces los arcos $d$-inducidos son arcos de mínima longitud respecto de la función distancia $d$, y en este caso, la función distancia $d$ pueden modelarse como un problema de cálculo de variaciones.
\end{abstract}

Descriptores: Funciones distancia generalizadas, longitud de arco, desigualdad del triángulo, métrica de Finsler.

\section{Abstract}

We propose a theoretical framework for modeling generalized distance functions, which can be asymmetric and non-positive definite. We give a definition of arc length associated to a generalized distance function $d$. Our distance function d satisfies the identity property but, unlike metrics, may not satisfy the triangle inequality, or symmetry and definiteness properties. We show that each distance function dinduces certain arcs, called "d-induced", which satisfy a conservation law of the distance $d$ and are a generalization of the straight line segments of the Euclidean space. We also show that if d satisfies the triangle inequality, then the d-induced arcs are arcs of minimal length with respect to the distance function $d$, and in this case, the distance function d can be modeled as a problem of calculus of variations.

Keywords: Generalized distance functions, arc length, triangle inequality, Finsler metric. 
DOI: http://dx.doi.org/10.22201/fi.25940732e.2008.09n4.026

Funciones distancia asimétricas y no positivas definidas Parte I: Marco teórico

\section{Introducción}

Frecuentemente, no es posible recabar los datos de las "distancias" entre todos los pares ordenados de puntos de una región dada, por lo que es útil contar con funciones distancia.

En este trabajo, se propone una definición de función distancia (generalizada), la cual permite generalizar el concepto de longitud de arco, y este a su vez, permite deducir la existencia de ciertos arcos que cumplen una ley de conservación de la distancia.

Se da una definición de función distancia generalizada, como una función binaria que cumple la propiedad de identidad (la distancia de un punto consigo mismo es cero), es decir, una función distancia generalizada puede no satisfacer la desigualdad del triángulo, simetría y no negatividad, propiedades requeridas por las métricas. Se define la longitud de arco asociado a una función distancia generalizada $d$, como la integral de la derivada direccional unilateral $F(\mathbf{x}, \mathbf{v})$ de $d$ a lo largo del arco. La función $F$ representa la razón de cambio de la función distancia en un punto dado $\mathbf{x}$ en la dirección considerada $\mathbf{v}$.

La propiedad de identidad es necesaria para que una función distancia $d$ determine arcos " $d$-inducidos", los cuales cumplen un principio de conservación de la $d$-distancia. Se definirá "premétrica", como una función distancia $d$, que además de la propiedad de identidad, cumple la desigualdad del triángulo. Se muestra que los ar$\cos d$-inducidos por una premétrica son arcos de mínima longitud, con respecto a la función distancia $d$. Se explora el problema de modelar una función distancia $d$, cuya derivada direccional unilateral es una función $F_{0}(\mathbf{x}, \mathbf{v})$ dada a priori, que llamamos función fundamental de $d$. En este problema, $d$ se obtiene resolviendo un problema de cálculo de variaciones. Se muestra además que si $F_{0}$ es una función convexa en la dirección $\mathbf{v}$, entonces $F_{0}$ coincide con la derivada direccional unilateral $F(\mathbf{x}, \mathbf{v})$ de $d$.

\section{Función distancia generalizada y longitud de un arco}

En esta sección se propone una definición de función distancia que puede no satisfacer la desigualdad del triángulo ni las condiciones de simetría y no negatividad, requeridas por las métricas. También una definición de longitud de arco asociada a una función distancia.

La función distancia generalizada $d$, o función distancia $d$, se define como una función binaria $d: R^{n} \times R^{n} \rightarrow R$ que cumple la propiedad de identidad (la distancia de un punto consigo mismo es cero, $\left.d(\mathbf{a}, \mathbf{a})=0, \forall \mathbf{a} \in R^{n}\right)$.

Para definir la longitud de arco asociada con una función distancia generalizada $d$ y obtener una expresión para determinarla, se dan las siguientes definiciones:

Un camino en $R^{n}$ de $\mathbf{a} \in R^{n}$ hasta $\mathbf{b} \in R^{n}$ es una función continua $\mathbf{x}:[a, b] \rightarrow R^{n}$ tal que $\mathbf{x}(a)=\mathbf{a}$ y $\mathbf{x}(b)=\mathbf{b}$. La imagen orientada $C(\mathbf{a}, \mathbf{b}) \subseteq R^{n}$ del camino $\mathbf{x}:[a, b] \rightarrow R^{n}$ se llama arco (orientado) de $\mathbf{a} \in R^{n}$ a $\mathbf{b} \in R^{n}$. En este trabajo, un arco significa un arco orientado. Un $\operatorname{arco} C(\mathbf{a}, \mathbf{b})$ se dice ser arco clase $C^{1}$ si tiene una representación paramétrica $\mathbf{x}:[a, b] \rightarrow R^{n}$ clase $C^{1}$, cuya derivada es diferente de cero en su dominio.

El conjunto de todos los arcos clase $C^{1}$ en $R^{n}$ se denota por $\Omega$. Por simplicidad, el conjunto de todos los arcos de clase $C^{1}$ que conectan a con $\mathbf{b}$, y el conjunto de todas las representaciones paramétricas de estos arcos, se denotan por $\Omega_{[\mathbf{a}, \mathbf{b}]}$.

Una sucesión de puntos en $R^{n}$ de la forma

$$
\left(\mathbf{a}=\mathbf{x}_{0}, \mathbf{x}_{1}, \mathbf{x}_{2}, \ldots, \mathbf{x}_{k}, \mathbf{x}_{k+1}=\mathbf{b}\right),
$$

donde $k \geq 1$, se dice que es una sucesión en $R^{n}$ desde $\mathbf{a}$ hasta $\mathbf{b}$. Los puntos $\mathbf{a}$ y $\mathbf{b}$ se llaman puntos extremos de la sucesión. El conjunto de todas las sucesiones en $R^{n}$ desde $\mathbf{a}$ hasta $\mathbf{b}$ se denota por $P[\mathbf{a}, \mathbf{b}]$.

Para cada sucesión de puntos en $\mathrm{R}^{n}$,

$$
P=\left(\mathbf{a}=\mathbf{x}_{0}, \mathbf{x}_{1}, \mathbf{x}_{2}, \ldots, \mathbf{x}_{k}, \mathbf{x}_{k+1}=\mathbf{b}\right),
$$

la función distancia $d: R^{n} \times R^{n} \rightarrow R$ determina un número real $\Lambda(P)$, que llamamos la $d$-longitud de la sucesión $P$ y está definida como la suma de las distancias respecto de la función distancia $d$ (suma de las $d$-distancias):

$$
\Lambda(P)=\sum_{i=0}^{k} d\left(\mathbf{x}_{i}, \mathbf{x}_{i+1}\right)
$$

para toda

$$
P=\left(\mathbf{a}=\mathbf{x}_{0}, \mathbf{x}_{1}, \mathbf{x}_{2}, \ldots \mathbf{x}_{k}, \mathbf{x}_{k+1}=\mathbf{b}\right) \in P[\mathbf{a}, \mathbf{b}]
$$

Consideramos una partición de un arco como una partición del arco en subarcos. Cada partición $P$ de una arco $C(\mathbf{a}, \mathbf{b})$ determina una sucesión $\left(\mathbf{a}=\mathbf{x}_{0}, \mathbf{x}_{1}, \mathbf{x}_{2}, \ldots, \mathbf{x}_{k}\right.$, $\left.\mathbf{x}_{k+1}=\mathbf{b}\right)$ de puntos de $C(\mathbf{a}, \mathbf{b})$. Recíprocamente, cada sucesión ( $\left.\mathbf{a}=\mathbf{x}_{0}, \mathbf{x}_{1}, \mathbf{x}_{2}, \ldots, \mathbf{x}_{k}, \mathbf{x}_{k+1}=\mathbf{b}\right)$ de puntos de $C(\mathbf{a}, \mathbf{b})$ determina la partición $P$. Para simplificar, se 
denota también por $P$ la sucesión de puntos de $C(\mathbf{a}, \mathbf{b})$ correspondiente a la partición $P$. La partición trivial de un $\operatorname{arco} C(\mathbf{a}, \mathbf{b})$ es el conjunto $\{C(\mathbf{a}, \mathbf{b})\}$, el cual está determinado por la sucesión $\left(\mathbf{a}=\mathbf{x}_{0}, \mathbf{x}_{1}=\mathbf{b}\right)$. Un refinamiento de una partición $P$ del $\operatorname{arco} C(\mathbf{a}, \mathbf{b})$ es una partición $Q$ de $C(\mathbf{a}, \mathbf{b})$, tal que, cada elemento de $Q$ está contenido en un elemento de $P$. El conjunto de todas las particiones de $C(\mathbf{a}, \mathbf{b})$ se denota por $P[C(\mathbf{a}, \mathbf{b})]$. Se define la $d$-longitud de una partición $P \in P[C(\mathbf{a}, \mathbf{b})]$ de $C(\mathbf{a}, \mathbf{b})$ como la $d$-longitud de la sucesión $P, \Lambda(P)$.

Se define la longitud de arco asociada con la función distancia generalizada $d$ de un arco $C$, o d-longitud de $C$, como un número real $L$ tal que, para cada $\varepsilon>0$, existe una partición $P_{\varepsilon}$ de $C$ tal que $|L-\Lambda(P)|<\varepsilon$ para todo refinamiento $P$ de $P_{\varepsilon}$. Si la $d$-longitud de un arco existe, entonces es única.

Si la $d$-longitud de $C(\mathbf{a}, \mathbf{b})$ es finita, entonces $C(\mathbf{a}, \mathbf{b})$ se dice $d$-rectificable y $l_{d}(C(\mathbf{a}, \mathbf{b}))$ denota la $d$-longitud de $C(\mathbf{a}, \mathbf{b})$. Es inmediato que los subarcos de cualquier partición $P$ de un arco $d$-rectificable $C(\mathbf{a}, \mathbf{b})$ son arcos $d$-rectificables, y que la suma de sus $d$-longitudes es igual a la $d$-longitud de $C(\mathbf{a}, \mathbf{b})$.

La derivada direccional unilateral de una función $\left.d(\mathbf{x},)^{\cdot}\right)$ en $\mathbf{x}$ en la dirección $\mathbf{v}$ se denota por $F(\mathbf{x}, \mathbf{v})$ y se define por

$$
F(\mathbf{x}, \mathbf{v})=\lim _{\Delta s \rightarrow 0^{+}} \frac{d(\mathbf{x}, \mathbf{x}+\Delta s \mathbf{v})-d(\mathbf{x}, \mathbf{x})}{\Delta s},
$$

(Rockafellar 1970). Debido a que la función distancia generalizada satisface la propiedad de identidad, $d(\mathbf{x}, \mathbf{x})=0, F$ se escribe como

$$
F(\mathbf{x}, \mathbf{v})=\lim _{\Delta_{s} \rightarrow 0^{+}} \frac{d(\mathbf{x}, \mathbf{x}+\Delta s \mathbf{v})}{\Delta s} \text { para toda } \mathbf{x}, \mathbf{v} \in R^{n}
$$

donde $F(\mathbf{x}, \mathbf{0})=0$ para todo $\mathbf{x} \in R^{n}$.

La función F: $R^{n} \times R^{n} \rightarrow R$ dada por (1) es la derivada direccional unilateral $F$ de la función distancia $d$. La función $F$ evaluada en un punto $\mathbf{x}$ y en una dirección $\mathbf{v}$ se denota por $F(\mathbf{x}, \mathbf{v})$, y $F$ a lo largo del camino $\mathbf{x}:[a, b] \rightarrow R^{n}$ se denota por $F(\mathbf{x}(\mathrm{s}), \dot{\mathbf{x}}(\mathrm{s}))$.

Ahora se va a determinar la $d$-longitud de un arco $C(\mathbf{a}, \mathbf{b})$ en términos de $F$, suponiendo que $C(\mathbf{a}, \mathbf{b})$ es clase $C^{1}$ y además $d$-rectificable.

Como se verá, esta última suposición resulta redundante. Sea $\mathbf{x}:[a, b] \rightarrow R^{n}$ una representación paramétrica clase $C^{1}$ de $C(\mathbf{a}, \mathbf{b})$.

Cualquier conjunto ordenado de puntos interiores de $[a, b], s_{1}, s_{2}, \ldots, s_{k} \in(a, b)$, con $k>0$, determina una partición no trivial $\left(a=s_{0}, s_{1}, s_{2}, \ldots, s_{k+1}=b\right)$ de $[a, b]$, y una partición no trivial $P=\left(\mathbf{a}=\mathbf{x}\left(s_{0}\right), \mathbf{x}\left(s_{1}\right), \mathbf{x}\left(s_{2}\right), \ldots\right.$, $\left.\mathbf{x}\left(s_{k}\right), \mathbf{x}\left(s_{k+1}\right)=\mathbf{b}\right)$ del arco $C(\mathbf{a}, \mathbf{b})$. Por tanto, la $d$-longitud de $P$ es

$$
\Lambda(P)=\sum_{i=0}^{k} d\left(\mathbf{x}\left(s_{i}\right), \mathbf{x}\left(s_{i+1}\right)\right) .
$$

Supóngase que $F(\mathbf{x}, \mathbf{v})$ es una función continua sobre su dominio. Puesto que $\mathbf{x}(t)$ y $\dot{\mathbf{x}}(t)$ son funciones continuas, entonces $F(\mathbf{x}(t), \dot{\mathbf{x}}(t))$, es una función continua y acotada en $[a, b]$ y por tanto, integrable. Debido a la existencia de $F(\mathbf{x}(t), \dot{\mathbf{x}}(t))$ en $t \in[a, b]$, para todo $\varepsilon>0$ existe $\Delta s>0$ tal que

$$
\left|F(\mathbf{x}(\xi), \dot{\mathbf{x}}(\xi))-\frac{d(\mathbf{x}(\xi), \mathbf{x}(\xi)+\dot{\mathbf{x}}(\xi) \Delta s)}{\Delta s}\right|<\varepsilon
$$

para toda $\xi \in[t, t+\Delta s]$.

Esto puede ser aplicado a una partición

$$
\left(a=s_{0}, s_{1}, s_{2}, \ldots, s_{k+1}=b\right) \text { de }[a, b] .
$$

Por tanto, para todo $\varepsilon>0$ existe a una partición $P_{\varepsilon}$ de $C(\mathbf{a}, \mathbf{b})$ tal que

$$
\left|F\left(\mathbf{x}\left(\xi_{i}\right), \dot{\mathbf{x}}\left(\xi_{i}\right)\right)-\frac{d\left(\mathbf{x}\left(\xi_{i}\right), \mathbf{x}\left(\xi_{i}\right)+\dot{\mathbf{x}}\left(\xi_{i}\right) \Delta s_{i}\right)}{\Delta s_{i}}\right|<\varepsilon
$$

para toda $\xi_{i} \in\left[s_{i}, s_{i+1}\right], i=0, \ldots, k$, para todo refinamiento $P$ de $P_{\varepsilon}$. Por tanto,

$$
\left|F\left(\mathbf{x}\left(\xi_{i}\right), \dot{\mathbf{x}}\left(\xi_{i}\right)\right) \Delta s_{i}-d\left(\mathbf{x}\left(\xi_{i}\right), \mathbf{x}\left(\xi_{i}\right)+\dot{\mathbf{x}}\left(\xi_{i}\right) \Delta s_{i}\right)\right|<
$$

$\varepsilon \Delta s_{i}$ para toda $\xi_{i} \in\left[s_{i}, s_{i+1}\right], i=0, \ldots, k$

se cumple. Puesto que

$$
\Lambda(P)=\sum_{i=0}^{k} d\left(\mathbf{x}\left(\xi_{i}\right), \mathbf{x}\left(\xi_{i}\right)+\dot{\mathbf{x}}\left(\xi_{i}\right) \Delta s_{i}\right),
$$

se obtiene

$$
\left|\sum_{i=0}^{k} F\left(\mathbf{x}\left(\xi_{i}\right), \dot{\mathbf{x}}\left(\xi_{i}\right)\right) \Delta s_{i}-\Lambda(P)\right|<\varepsilon(b-a)
$$

para toda $\xi_{i} \in\left[s_{i}, s_{i+1}\right)$. 
La última condición es también válida si se reemplaza $\varepsilon(b-a)$ por $\varepsilon / 2$. Puesto que se supone que $C(\mathbf{a}, \mathbf{b})$ es un arco $d$-rectificable, para todo $\varepsilon>0$ existe una partición $P_{\varepsilon}$ de $C(\mathbf{a}, \mathbf{b})$ tal que

$$
\left|\sum_{i=0}^{k} F\left(\mathbf{x}\left(\xi_{i}\right), \dot{\mathbf{x}}\left(\xi_{i}\right)\right) \Delta s_{i}-\Lambda(P)\right|<\frac{\varepsilon}{2}
$$

para toda $\xi_{i} \in\left[s_{i}, s_{i+1}\right) ; \mathrm{y}$

$$
|L-\Lambda(P)|<\frac{\varepsilon}{2}
$$

se satisfacen para todo refinamiento $P$ de $P_{\varepsilon}$.

Por tanto, para toda $\varepsilon>0$, existe una partición $P_{\varepsilon}$ de $C(\mathbf{a}, \mathbf{b})$ tal que

$$
\left|L-\sum_{i=0}^{k} F\left(\mathbf{x}\left(\xi_{i}\right), \dot{\mathbf{x}}\left(\xi_{i}\right)\right) \Delta s_{i}\right|<\varepsilon, \text { para toda } \xi_{i} \in\left[s_{i}, s_{i+1}\right),
$$

para todo refinamiento $P$ de $P_{\varepsilon}$. Por tanto,

$$
L=\int_{a}^{b} F(\mathbf{x}(s), \dot{\mathbf{x}}(s)) d s
$$

(Sagan, 1974). Para todo arco $C(\mathbf{a}, \mathbf{b})$ clase $C^{1}$, el integrando anterior, $F(\mathbf{x}(s), \dot{\mathbf{x}}(s))$, es una función continua en $[a, b]$, y por tanto $L$ está bien definida y $C(\mathbf{a}, \mathbf{b})$ es $d$-rectificable.

Por tanto, la suposición de que $C(\mathbf{a}, \mathbf{b})$ es $d$-rectificable resulta redundante. Entonces se ha demostrado el siguiente teorema:

Teorema 1

(Determinación de la $d$-longitud de un arco $d$-rectificable)

Si la función distancia $d: R^{n} \times R^{n} \rightarrow R$ tiene una derivada direccional unilateral continua, $F: R^{n} \times R^{n} \rightarrow R$, entonces todo arco $C(\mathbf{a}, \mathbf{b})$ clase $C^{1}$ es $d$-rectificable y su $d$-longitud está dada por

$$
l_{d}(C(\mathbf{a}, \mathbf{b}))=\int_{a}^{b} F(\mathbf{x}(s), \dot{\mathbf{x}}(s)) d s
$$

donde $\mathbf{x}:[a, b] \rightarrow R^{n}$ es una representación paramétrica clase $C^{1}$ de $C(\mathbf{a}, \mathbf{b})$.

Q.E.D
El siguiente teorema establece propiedades importantes de la derivada direccional unilateral $F$ de una función distancia $d$.

Estas propiedades son útiles en el modelado de funciones distancia.

\section{Teorema 2}

(Propiedades de la derivada direccional unilateral $F$ de una función distancia $d$ )

Sea $d: R^{n} \times R^{n} \rightarrow R$ una función distancia y $F$ la derivada direccional unilateral de $d$, dada por (1). Entonces se tiene:

a) $F(\mathbf{x}, \mathbf{0})=0$ para todo $\mathbf{x} \in R^{n}$.

b) La función $F$ es positivamente homogénea de grado uno: $F(\mathbf{x}, \alpha \mathbf{v})=\alpha F(\mathbf{x}, \mathbf{v})$ para toda $\alpha>0$.

c) Para $n \geq 2, F(\mathbf{x}(s), \dot{\mathbf{x}}(s))$ no depende explícitamente del parámetro $s$ del camino.

d) La $d$-longitud de un arco $d$-rectificable no depende de su representación paramétrica.

e) Si $d$ cumple la desigualdad del triángulo, entonces $F(\mathbf{x}, \mathbf{v})$ es una función convexa en $\mathbf{v}$ para cada $\mathbf{x}$ : $F(\mathbf{x}, \alpha \mathbf{v}+(1-\alpha) \mathbf{w}) \leq \alpha F(\mathbf{x}, \mathbf{v})+(1-\alpha) F(\mathbf{x}, \mathbf{w})$ para toda $\alpha \in[0,1], \mathbf{x} \in R^{n}$.

\section{Demostración}

a) Inmediato de la definición de $F$ y de que $d(\mathbf{x}, \mathbf{x})=0$ para todo $\mathbf{x} \in R^{n}$.

b) Para $\alpha>0$,

$$
\begin{aligned}
& F(\mathbf{x}, \alpha \mathbf{v})=\lim _{h \rightarrow 0^{+}} \frac{d(\mathbf{x}, \mathbf{x}+\alpha h \mathbf{v})}{h}= \\
& \alpha \lim _{h \rightarrow 0^{+}} \frac{d(\mathbf{x}, \mathbf{x}+\alpha h \mathbf{v})}{\alpha h}=\alpha \lim _{\beta \rightarrow 0^{+}} \frac{d(\mathbf{x}, \mathbf{x}+\beta \mathbf{v})}{\beta}=\alpha F(\mathbf{x}, \mathbf{v}) .
\end{aligned}
$$

c) La derivada direccional unilateral $F$ a lo largo del camino $\mathbf{x}:[a, b] \rightarrow R^{n}$, dada por

$F(\mathbf{x}(s), \dot{\mathbf{x}}(s))=\lim _{\Delta s \rightarrow 0^{+}} \frac{d(\mathbf{x},(s), \mathbf{x}(s)+\dot{\mathbf{x}}(s) \Delta s)}{\Delta s}$,

no depende explícitamente de $s$. 
d) La $d$-longitud de un arco $d$-rectificable está dada por (2). Sea $s=s(t)$ una transformación de $\mathbf{x}(t)$ que preserva la orientación. Por tanto $d s / d t>0$ y $s(t)$ es una función invertible. La homogeneidad positiva de $F$ y $d s / d t>0$ implican que

$$
\begin{aligned}
& F(\mathbf{x}, d \mathbf{x}(t) / d t) d t=F(\mathbf{x}, \dot{\mathbf{x}} d s / d t) d t= \\
& F(\mathbf{x}, \dot{\mathbf{x}})(d s / d t) d t=F(\mathbf{x}, \dot{\mathbf{x}}) d s .
\end{aligned}
$$

Puesto que $F$ no depende explícitamente del parámetro $s$, entonces $F(\mathbf{x}(s), \dot{\mathbf{x}}(s)) d s$ es invariante bajo la transformación $s=s(t)$.

e) De la ecuación (1) y por el inciso (b),

$$
\begin{aligned}
& F(\mathbf{x}, \alpha \mathbf{v}+(1-\alpha) \mathbf{w})=\lim _{h \rightarrow 0^{+}} \frac{d(\mathbf{x}, \mathbf{x}+(\alpha \mathbf{v}+(1-\alpha) \mathbf{w}) h)}{h} \\
& \leq \lim _{h \rightarrow 0^{+}} \frac{d(\mathbf{x}, \mathbf{x}+\alpha \mathbf{v} h)+d(\mathbf{x}+\alpha \mathbf{v} h, \mathbf{x}+\alpha \mathbf{v} h+(1-\alpha) \mathbf{w} h)}{h} \\
& =F(\mathbf{x}, \alpha \mathbf{v})+F(\mathbf{x},(1-\alpha) \mathbf{w})=\alpha F(\mathbf{x}, \mathbf{v})+(1-\alpha) F(\mathbf{x}, \mathbf{w})
\end{aligned}
$$

Q.E.D.

\section{Arcos inducidos por una función distancia $d$}

Nuestra definición de $d$-longitud de arco permite deducir la existencia de ciertos arcos caracterizados por la función distancia $d$, que les llamamos arcos $d$-inducidos. En esta sección definimos arco inducido por una función distancia $d$.

Sea $d: R^{n} \times R^{n} \rightarrow R$ una función distancia. Decimos que un $\operatorname{arco} C(\mathbf{a}, \mathbf{b})$ es $d$-inducido si todas sus particiones tienen la misma $d$-longitud.

Por la definición dada de longitud de arco, todo arco $d$-inducido $C(\mathbf{a}, \mathbf{b})$ es $d$ - rectificable, y su $d$-longitud es igual a la $d$-longitud de su partición trivial, $l_{d}(C(\mathbf{a}, \mathbf{b}))=$ $d(\mathbf{a}, \mathbf{b})$.

Es inmediato que cada subarco de un arco $d$-inducido es un arco $d$-inducido.

Equivalentemente, $C(\mathbf{a}, \mathbf{b})$ es un arco $d$-inducido si la $d$-longitud de toda partición de $C(\mathbf{a}, \mathbf{b}), P=\left(\mathbf{a}=\mathbf{x}_{0}, \mathbf{x}_{1}\right.$, $\mathbf{x}_{2}, \ldots, \mathbf{x}_{k}, \mathbf{x}_{k+1}=\mathbf{b}$ ), con $k \geq 0$, es igual a la $d$-distancia de $\mathbf{a}$ a $\mathbf{b}$,

$$
\begin{aligned}
& d(\mathbf{a}, \mathbf{b})=\sum_{i=0}^{k} d\left(\mathbf{x}_{i}, \mathbf{x}_{i+1}\right), \text { para toda } \\
& P=\left(\mathbf{a}, \mathbf{x}_{1}, \ldots, \mathbf{x}_{k} \mathbf{b}\right) \in P[C(\mathbf{a}, \mathbf{b})],
\end{aligned}
$$

la cual es la $d$-longitud de la partición trivial de $C(\mathbf{a}, \mathbf{b})$. Es decir, un arco de $\mathbf{a}$ a $\mathbf{b}$ es $d$-inducido si, y sólo si, la $d$-distancia de $\mathbf{a}$ a $\mathbf{b}$ es igual a la suma de las $d$-distancias entre todos los puntos consecutivos de cualquier sucesión de puntos en el arco, donde la sucesión incluye los puntos extremos $\mathbf{a} \mathbf{y} \mathbf{b}$.

Es inmediato que un arco $C(\mathbf{a}, \mathbf{b})$ es $d$-inducido si, $y$ sólo si, la restricción de $d$ a $C(\mathbf{a}, \mathbf{b})$ cumple la igualdad del triángulo respecto del punto final $\mathbf{b}$,

$$
d(\mathbf{x}(s), \mathbf{b})=d(\mathbf{x}(s), \mathbf{x}(t))+d(\mathbf{x}(t), \mathbf{b}) \quad a \leq s \leq t \leq b,
$$

donde $\mathbf{x}:[a, b] \rightarrow R^{n}$ es una representación paramétrica $\operatorname{de} C(\mathbf{a}, \mathbf{b})$.

\section{Premétricas y arcos mínimos}

Si una función distancia no satisface la desigualdad del triángulo, los arcos mínimos no necesariamente son arcos inducidos y el concepto tradicional de que la distancia desde un punto hasta otro es igual a la longitud del arco "más corto" que los conecta, no es válido. En esta sección definimos premétrica, la cual es una función distancia que cumple la desigualdad del triángulo, y se dan dos teoremas importantes para el modelado de premétricas.

Esto es de interés porque, en general, las funciones distancia que intervienen en el modelado de problemas de la vida real cumplen la desigualdad del triángulo; en estos casos, los arcos mínimos sí coinciden con los arcos inducidos y se pueden determinar mediante un problema de cálculo variacional.

Una función distancia $d$ que cumple la desigualdad del triángulo (para toda $\mathbf{a}, \mathbf{b}, \mathbf{c} \in R^{n} d(\mathbf{a}, \mathbf{b}) \leq d(\mathbf{a}, \mathbf{c})+d$ (c, b)) la llamamos premétrica.

Una función distancia es completa si todo par ordenado de puntos $\mathbf{a}, \mathbf{b}$ en $R^{n}$ está conectado por un $\operatorname{arco} d$-inducido.

Si la función distancia $d$ es completa y cumple la desigualdad del triángulo, es decir, $d$ es una premétrica completa, entonces los arcos $d$-inducidos son arcos de mínima $d$-longitud, y la función distancia $d$ está determinada por su derivada direccional unilateral $F$.

La última afirmación implica que $d$ y $F$ contienen la "misma información", donde $d$ proporciona "información global", ya que su valor depende de dos puntos localizados arbitrariamente en $R^{n}$, y $F$ proporciona "información local" porque su valor depende del punto y la dirección considerados. 
Es inmediato que una función distancia $d: R^{n} \times R^{n} \rightarrow R$ satisface la desigualdad del triángulo, $d(\mathbf{a}, \mathbf{b}) \leq d(\mathbf{a}, \mathbf{c})+$ $d(\mathbf{c}, \mathbf{b})$ para toda $\mathbf{a}, \mathbf{b} \in R^{n}$, si y sólo si, para toda sucesión $P=\left(\mathbf{a}=\mathbf{x}_{0}, \mathbf{x}_{1}, \mathbf{x}_{2}, \ldots, \mathbf{x}_{k}, \mathbf{x}_{k+1}=\mathbf{b}\right)$ la d-distancia entre los puntos extremos $P$ es menor o igual que la $d$-longitud de la sucesión de $P$, es decir, si y sólo si

$$
d(\mathbf{a}, \mathbf{b}) \leq \sum_{i=0}^{k} d\left(\mathbf{x}_{i}, \mathbf{x}_{i+1}\right)
$$

para toda $P=\left(\mathbf{a}=\mathbf{x}_{0}, \mathbf{x}_{1}, \mathbf{x}_{2}, \ldots, \mathbf{x}_{k}, \mathbf{x}_{k+1}=\mathbf{b}\right)$.

Sea $d: R^{n} \times R^{n} \rightarrow R$ una función distancia tal que su derivada direccional unilateral $F$ es una función continua. Si $\mathbf{x}:[a, b] \rightarrow R^{n}$ es un camino clase $C^{1}$ de $\mathbf{a}$ a $\mathbf{b}$ que resuelve

$$
\min _{x \in \Omega_{a, b]}} \int_{a}^{b} F(\mathbf{x}(s), \dot{\mathbf{x}}(s)) d s \text { para toda } \mathbf{a}, \mathbf{b} \in R^{n},
$$

entonces se dice que la imagen de $\mathbf{x}, C(\mathbf{a}, \mathbf{b}) \in \Omega_{[\mathbf{a}, \mathbf{b}]}$, es un arco de minima $d$-longitud o arco d-minimal. Por las propiedades de las integrales, todos los subarcos de un $\operatorname{arco} d$-minimal son arcos $d$-minimales.

\section{Teorema 3}

(Para cualquier premétrica los arcos $d$-inducidos son arcos $d$-minimales)

Si $d: R^{n} \times R^{n} \rightarrow R$ es una premétrica con una derivada direccional unilateral $F(\mathbf{x}, \mathbf{v})$ continua en $\mathbf{x}$ para cada $\mathbf{v} \in R^{n}$, entonces:

a) $F: R^{n} \times R^{n} \rightarrow R$ es continua, y por tanto, la longitud de arco de $C(\mathbf{a}, \mathbf{b})$ está dada por (2);

b) Si $P, Q \in P[C(\mathbf{a}, \mathbf{b})]$ son dos particiones de un arco $C(\mathbf{a}, \mathbf{b})$, tales que $P$ es un refinamiento de $Q$, entonces $\Lambda(Q) \leq \Lambda(P)$;

c) Para todo arco $C(\mathbf{a}, \mathbf{b})$ clase $C^{1}, l_{d}(C(\mathbf{a}, \mathbf{b}))=\sup \{\Lambda(P)$ : $P \in P[C(\mathbf{a}, \mathbf{b})]\}$

d) $(\mathbf{a}, \mathbf{b}) \leq \Lambda(P) \leq l_{d}(C(\mathbf{a}, \mathbf{b}))$ para toda partición $P \in P$ $[C(\mathbf{a}, \mathbf{b})]$ de un arco $d$-rectificable clase $C^{1}$;

e) Para todo arco $C(\mathbf{a}, \mathbf{b})$ clase $C^{1}, d(\mathbf{a}, \mathbf{b})=l_{d}(C(\mathbf{a}, \mathbf{b}))$ si y sólo si $C(\mathbf{a}, \mathbf{b})$ es un arco $d$-inducido;

f) Todo arco $d$-inducido es un arco $d$-minimal; g) Si $d$ es completa, entonces

$$
d(\mathbf{a}, \mathbf{b})=\min _{x \in \Omega_{[a, b]}} \int_{a}^{b} F(\mathbf{x}(s), \dot{\mathbf{x}}(s)) d s, \text { para toda } \mathbf{a}, \mathbf{b} \in R^{n}
$$

donde el mínimo se alcanza para un arco $d$-inducido de $\mathbf{a}$ a $\mathbf{b}$.

\section{Demostración}

a) $d$ cumple la desigualdad del triángulo y, por (e) del teorema 2, $F(\mathbf{x}, \mathbf{v})$ es una función convexa en $\mathbf{v}$ para cada $\mathbf{x} \in R^{n}$. Por tanto, $F$ es una función continua (Rockafellar, 1970).

b) Se puede probar directamente de la desigualdad del triángulo.

c) Sea $C(\mathbf{a}, \mathbf{b})$ un arco clase $C^{1}$. Entonces $C(\mathbf{a}, \mathbf{b})$ es $d$-rectificable con una longitud que denotamos por $L$. Por tanto, para todo $\varepsilon>0$ existe una partición $P_{\varepsilon}$ de $C(\mathbf{a}, \mathbf{b})$ tal que $|L-\Lambda(P)|<\varepsilon$ para todo refinamiento $P$ de $P_{\varepsilon}$. En particular, $\left|L-\Lambda\left(P_{\varepsilon}\right)\right|<\varepsilon$. Se tienen dos casos, $L \geq \Lambda\left(P_{\varepsilon}\right)$, o bien, $L<\Lambda\left(P_{\varepsilon}\right)$. El segundo caso se descarta porque si $C(\mathbf{a}, \mathbf{b})$ no es un arco $d$-inducido se llega a una contradicción: existe una $\varepsilon>0$ y una partición $Q$ de $C(\mathbf{a}, \mathbf{b})$ (la partición trivial, por ejemplo), de la cual $P_{\varepsilon}$ es un refinamiento y tal que $\Lambda(Q)-L>\varepsilon$; esto lleva a $\Lambda\left(P_{\varepsilon}\right)<\varepsilon+L<\Lambda(Q)$, en donde $(b)$ contradice que $P_{\varepsilon}$ es una partición de $Q$. Por tanto, si $d$ es una premétrica, entonces $\Lambda(P) \leq L$ para toda partición $P \in P[C(\mathbf{a}, \mathbf{b})]$ de $C(\mathbf{a}, \mathbf{b})$, y la longitud de un arco es $L$ si para todo $\varepsilon>0$ existe una partición $P_{\varepsilon} \operatorname{de} C(\mathbf{a}, \mathbf{b})$ tal que $L-\Lambda(P)<\varepsilon$ para todo refinamiento $P$ de $P_{\varepsilon}$. Por tanto, $L=\sup \{\Lambda(P)$ : $P \in P[C(\mathbf{a}, \mathbf{b})]\}$.

d) Cualquier partición de $C(\mathbf{a}, \mathbf{b})$ es un refinamiento de la partición trivial; por $(\mathrm{b}) d(\mathbf{a}, \mathbf{b}) \leq \Lambda(P)$. Por $\Lambda(\mathrm{c})$, $(P) \leq l_{d}(C(\mathbf{a}, \mathbf{b}))$.

e) Sea $C(\mathbf{a}, \mathbf{b})$ clase $C^{1}$. Si $C(\mathbf{a}, \mathbf{b})$ es un arco $d$-inducido, entonces $d(\mathbf{a}, \mathbf{b})=l_{d}(C(\mathbf{a}, \mathbf{b}))$. Recíprocamente, si $d(\mathbf{a}, \mathbf{b})=l_{d}(C(\mathbf{a}, \mathbf{b}))$, entonces por $(\mathrm{d})$ se tiene $d(\mathbf{a}, \mathbf{b})=$ $\Lambda(P)$ para toda $P \in P[C(\mathbf{a}, \mathbf{b})]$, y por tanto, $C(\mathbf{a}, \mathbf{b})$ es un arco $d$-inducido.

f) Si $C(\mathbf{a}, \mathbf{b})$ un arco $d$-inducido que no es un arco $d$-minimal, entonces $d(\mathbf{a}, \mathbf{b})=l_{d}(C(\mathbf{a}, \mathbf{b}))$ y $l_{d}\left(C^{*}\right.$ $(\mathbf{a}, \mathbf{b}))<l_{d}(C(\mathbf{a}, \mathbf{b}))$ para algún $\operatorname{arco} C^{*}(\mathbf{a}, \mathbf{b})$. Entonces se obtiene $l_{d}\left(C^{*}(\mathbf{a}, \mathbf{b})\right)<d(\mathbf{a}, \mathbf{b})$, lo que contradice $(\mathrm{d})$. 
DOI: http://dx.doi.org/10.22201/fi.25940732e.2008.09n4.026

H. Sánchez-Larios y S.T. Guillén-Burguete

Por tanto, todo arco $d$-inducido es un arco $d$-minimal.

g) $d$ completa implica que para todo par ordenado de puntos $\mathbf{a}$ y $\mathbf{b}$ existe un arco $d$-inducido $C(\mathbf{a}, \mathbf{b})$ que los conecta, el cual por (e) tiene $d$-longitud $d(\mathbf{a}, \mathbf{b})$. Por (f), $C(\mathbf{a}, \mathbf{b})$ es un arco $d$-minimal, y por lo que resuelve (3).

Q.E.D.

Por el teorema 1, la longitud de un arco respecto de una función distancia $d$ es la integral (2) de la derivada direccional unilateral $F(\mathbf{x}, \mathbf{v})$ de $d$ a lo largo del arco. Exploramos ahora la relación recíproca entre las funciones $F$ y $d$ : Sea $d: R^{n} \times R^{n} \rightarrow R$ la función distancia definida por

$$
d(\mathbf{a}, \mathbf{b})=\min _{x \in \Omega_{[a, b]}} \int_{a}^{b} F_{0}(\mathbf{x}(s), \dot{\mathbf{x}}(s)) d s, \text { para toda } \mathbf{a}, \mathbf{b} \in R^{n}
$$

donde $F_{0}: R^{n} \times R^{n} \rightarrow R$ es una función dada a priori que satisface las condiciones (a)-(c) del teorema 2, llamada función fundamental de $d$. El problema de minimización (4) se denomina problema variacional correspondiente a la función $F_{0}$.

Interesa ahora saber qué condiciones adicionales debe cumplir $F_{0}$ para que ésta sea la derivada direccional unilateral de $d$ dada por (1). En primer lugar, para que la función $d$ esté bien definida, $F_{0}$ debe ser tal que el problema de cálculo de variaciones (4) tenga una solución para cada par ordenado $\mathbf{a}, \mathbf{b} \in R^{n}$.

En segundo lugar, por las propiedades de las integrales de línea, la función $d$ dada por (4), cumple la desigualdad del triángulo, y por tanto, según (e) del teorema $2, F_{0}$ debe ser una función convexa con respecto a la variable dirección.

Ya sea que $F=F_{0} \circ F \neq F_{0}$, se cumple que

$d(\mathbf{a}, \mathbf{b})=\min _{x \in \Omega_{[a, b]}} \int_{a}^{b} F_{0}(\mathbf{x}(s), \dot{\mathbf{x}}(s)) d s=\min _{x \in \Omega_{[a, b]}} \int_{a}^{b} F(\mathbf{x}(s), \dot{\mathbf{x}}(s)) d s$.

Tenemos entonces el siguiente teorema.

Teorema 4

(Premétrica definida a partir de una función fundamental)

Sea $F_{0}: R^{n} \times R^{n} \rightarrow R$ una función positivamente homogénea de grado uno con $F_{0}(\mathbf{x}, \mathbf{0})=0$ para todo $\mathbf{x} \in R^{n}$, y tal que cumple la siguiente condición de solubilidad: para cada par ordenado $\mathbf{a}, \mathbf{b} \in \mathrm{R}^{n}$ existe un camino $\mathbf{x}:[a, b] \rightarrow R^{n}$ de $\mathbf{a}$ a $\mathbf{b}$ clase $C^{1}$ que resuelve el problema de cálculo de variaciones

$$
\begin{aligned}
& \min _{x \in \Omega_{[a, b]}} \int_{a}^{b} F_{0}(\mathbf{x}(s), \dot{\mathbf{x}}(s)) d s . \\
& \text { Sea } d: R^{n} \times R^{n} \rightarrow R \text { la función dada por (3). Entonces: }
\end{aligned}
$$

a) $d$ es una premétrica sobre $R^{n}$, la cual es completa si $F_{0}$ es la derivada direccional unilateral de $d$.

b) $F_{0}$ es la derivada direccional unilateral de $d$ si y solamente si, $F_{0}(\mathbf{x}, \mathbf{v})$ es convexa en $\mathbf{v}$.

\section{Demostración}

La homogeneidad positiva de $F_{0}$ implica que toda transformación continua que preserva la orientación de un camino $\mathbf{x}:[a, b] \rightarrow R^{n}$ que resuelve (4) es un camino que también resuelve (4), teniendo ambos caminos la misma imagen.

Por tanto, dados $\mathbf{a}$ y $\mathbf{b}$, cada solución de (4) depende sólo del arco y no de la elección particular de su representación paramétrica. Entonces, la función $d$ dada por (3) está bien definida.

a) Por las propiedades de las integrales, la función $d$ dada por (4) cumple la propiedad de identidad y la desigualdad del triángulo, y por tanto $d$ es una premétrica. Si $F=F_{0}$, entonces para cada par ordenado de puntos $\mathbf{a}$ y $\mathbf{b}$ existe un $\operatorname{arco} C(\mathbf{a}, \mathbf{b})$ que cumple $d(\mathbf{a}, \mathbf{b})=l_{d}(C(\mathbf{a}, \mathbf{b}))$, y por (e) del teorema 3 , $C(\mathbf{a}, \mathbf{b})$ es un arco $d$-inducido. Así que $d$ es una premétrica completa.

b) La homogeneidad positiva de $F_{0}$ implica que la convexidad de $F_{0}$ se reduce a:

$F_{0}\left(\mathbf{x}, \mathbf{v}_{1}+\mathbf{v}_{2}\right) \leq F_{0}\left(\mathbf{x}, \mathbf{v}_{1}\right)+F_{0}\left(\mathbf{x}, \mathbf{v}_{2}\right)$ para todo $\mathbf{v}_{1}, \mathbf{v}_{2} \in R^{n}$. Por tanto, la convexidad de $F_{0}$ implica que la derivada direccional unilateral $F$ de la función $d$ dada por (1) es la función $F_{0}$ :

$$
F(\mathbf{x}, \mathbf{v})=\lim _{h \rightarrow 0^{+}}\left(\frac{1}{h} \min _{x \in \Omega_{x, x+v h]}} \int_{x}^{x+v h} F_{0}(\mathbf{x}(s), \dot{\mathbf{x}}(s)) d s\right)=F_{0}(\mathbf{x}, \mathbf{v}) .
$$

La última igualdad se puede explicar como sigue. En el límite cuando $h \rightarrow 0^{+}, \mathbf{x}(s)$ se puede considerar constante, y por tanto, el integrando $F_{0}(\mathbf{x}(s), \dot{\mathbf{x}}(s))$ sólo depende de $\dot{\mathbf{x}}(s)$. Debido a la convexidad de $F_{0}$, la integral alcanza su valor mínimo si $\dot{\mathbf{x}}(s)$ tiene la dirección de $\mathbf{v}$ en todos los puntos a lo largo del arco que va de $\mathbf{x}$ a $\mathbf{x}+\mathbf{v} h$. Por 
tanto, el integrando $F_{0}(\mathbf{x}(s), \dot{\mathbf{x}}(s))$ permanece constante a lo largo del arco que va de $\mathbf{x}$ a $\mathbf{x}+\mathbf{v} h$ y toma el valor $F_{0}(\mathbf{x}, \mathbf{v})$. Para demostrar la afirmación recíproca, supóngase que la función $F_{0}$ que define a $d$ a través de (4) es igual a la derivada direccional unilateral de $d$. Por (a) $d$ es una premétrica, es decir, $d$ cumple la desigualdad del triángulo, y por (e) del teorema $2, F_{0}$ es una función convexa.

Q.E.D

El teorema 3 expresa que para toda premétrica con derivada direccional unilateral continua, la distancia de un punto a otro es igual a la $d$-longitud del arco más corto que los conecta, y el teorema 4 establece que si la $d$-distancia desde un punto hasta otro se define como "el mínimo de la longitud de los arcos que los conectan", entonces la función distancia resultante es una premétrica.

Cabe mencionar que en la métrica de Finsler, las condiciones sobre la función fundamental $F_{0}$ son más restrictivas que en el teorema 4 , pide que $F_{0}(\mathbf{x}, \mathbf{v})$ sea estrictamente convexa, no negativa y suave sobre su dominio (Anastasiei, 2004 y Chern, 2005).

\section{Conclusiones}

Se propone una definición de función distancia generalizada como una función binaria que cumple la propiedad de identidad, pero que no requiere satisfacer la desigualdad del triángulo ni las propiedades de simetría y no negatividad requeridas por las métricas, como es el caso de las métricas Lp y sus combinaciones lineales positivas usadas tradicionalmente en el modelado de funciones distancia. También se definió la longitud de arco, la cual permite asociar longitudes de arco a funciones distancia generalizadas. Se encontró que toda función distancia generalizada $d$ determina ciertos arcos, los cuales satisfacen una ley de conservación de las d-distancias. Se demostró que para cualquier premétrica (función distancia que cumple la desigualdad del triángulo), la distancia desde un punto hasta otro se puede expresar como el mínimo de la integral de línea de su derivada direccional unilateral para los caminos que unen dichos puntos. Esta relación entre las premétricas y sus derivadas direccionales unilaterales asegura que cualquier premétrica se puede definir por su derivada direccional unilateral a través de un problema de cálculo de variaciones.

\section{Referencias}

Anastasiei M. Finsler Vector Bundles-Metrizable Connections. Periodica Mathematica Hun- garica, 48(1-2):83-91. 2004.

Chern S. and Shen Z. Riemann-Finsler Geometry. World Scientific. 2005. Pp 1.

Rockafellar T. Convex Analysis. Princeton University Press. 1970. Pp. 213.

Sagan H. Advanced Calculus of Real-Valued Functions of a Real Variable and Vector-Valued Functions of a Vector Variable. Houghton Mifflin Company. Boston. 1974. Pp. 160.

\section{Semblanza de los autores}

Hérica Sánchez-Larios. Realizó la maestría y el doctorado en ingeniería (Investigación de operaciones), ambos en la Facultad de Ingeniería de la UNAM. Trabajó en PEMEX, fue instructora de cursos sobre instrumentación de laboratorios en CONACYT, y ha impartido clases en diversas universidades. Actualmente es investigadora del Instituto de Ingeniería de la UNAM y profesora en el Programa de Maestría y Doctorado en Ingeniería de la UNAM.

Servio Tulio Guillén-Burguete. Es egresado de la ESIME del IPN. Obtuvo la maestría en ingeniería de control y el doctorado en ingeniería (Investigación de operaciones), ambos en la Facultad de Ingeniería de la UNAM. Laboró en el Sistema de Transporte Colectivo Metro de la Ciudad de México. Es investigador del Instituto de Ingeniería de la UNAM, y profesor en el Programa de Maestría y Doctorado en Ingeniería, así como en la Facultad de Ciencias de la UNAM. 